TRANSACTIONS OF THE

AMERICAN MATHEMATICAL SOCIETY

Volume 365, Number 4, April 2013, Pages 1957-1975

S 0002-9947(2012)05757-6

Article electronically published on September 18, 2012

\title{
TRACKING A MOVING POINT IN THE PLANE
}

\author{
FREDERICK P. GARDINER AND NIKOLA LAKIC
}

Abstract. The Teichmüller theory of any hyperbolic Riemann surface $R$ induces two closely related metrics on $R$ in the following way. From a theorem of Bers, the fiber

$$
\mathbb{K}=\Psi^{-1}([\text { identity }])
$$

of the forgetful map $\Psi$ from the Teichmüller space Teich $(R-p)$ onto the Teichmüller space Teich $(R)$ is conformal to a disc and the evaluation map $\mathbb{K} \ni[f] \mapsto f(p) \in R$ is a universal covering of $R$. There are two infinitesimal metrics on $\mathbb{K}$ coming from Kobayashi's construction:

(1) Teich $_{\mathbb{K}}$ is the restriction of the Teichmüller infinitesimal metric on Teich $(R-p)$ to the submanifold $\mathbb{K}$, and

(2) $K o b_{\mathbb{K}}$ is the Kobayashi metric on $\mathbb{K}$.

We show these metrics, respectively, are the lifts via the evaluation map of infinitesimal forms $\lambda$ and $\rho$ on $R$, where $\lambda$ and $\rho$ are the Teichmüller and Poincaré densities. $\lambda$ and $\rho$ have very different descriptions. For plane domains

$$
\lambda(p)=\inf \left\{\|\bar{\partial} V\|_{\infty}\right\},
$$

where the infimum is taken over all continuous functions $V$ for which $V(p)=1$ and $V$ vanishes on the boundary of $R$, and

$$
\rho(p)=\inf \left\{1 /\left|f^{\prime}(0)\right|\right\},
$$

where the infimum is taken over all holomorphic functions $f$ mapping the unit disc into $R$ with $f(0)=p$. We also show

$$
\begin{gathered}
(1 / 2) K o b_{\mathbb{K}} \leq T e i c h_{\mathbb{K}} \leq K o b_{\mathbb{K}} \text { and } \\
(1 / 2) \rho \leq \lambda \leq \rho,
\end{gathered}
$$

and $\lambda / \rho=1 / 2$ when $R$ is simply connected, $\lambda / \rho=1$ when $R$ is a thrice punctured sphere, and in all other cases these inequalities are strict.

\section{INTRODUCTION}

A small movement of a point $p$ to another point on any hyperbolic Riemann surface $R$ changes the conformal structure of $R \backslash\{p\}$ but not the conformal structure of $R$. This idea can be expressed formally by observing that there is a natural projection between Teichmüller spaces,

$$
\Psi: T(R \backslash\{p\}) \rightarrow T(R) .
$$

Those deformations represented by points in $T(R \backslash\{p\})$ that do not alter the corresponding points in $T(R)$ are realized by homotopy classes of motions of $p$ on $R$. The map $\Psi$ is called the forgetful map because, for any deformation in $T(R \backslash\{p\})$, it forgets the motion of $p$ and remembers only how $R$ was deformed. The fiber $\mathbb{K}=\Psi^{-1}([i d])$ consists of the deformations caused by moving $p$ in $R$ while leaving

Received by the editors November 10, 2009 and, in revised form, July 20, 2011.

2010 Mathematics Subject Classification. Primary 30F60; Secondary 32G15, 30C70, 30C75.

The second author was partially supported by NSF grant 0700052 . 
the marked conformal structure of $R$ fixed. This fiber is the main topic of study by Bers in 3 . where he shows that when $R$ is of finite analytic type, $\mathbb{K}$ is conformal to the universal covering of $R$. Then $T(R \backslash\{p\})$ factored by the natural realization of the fundamental group of $R$ as a subgroup of the mapping class group of $R \backslash\{p\}$ is the universal curve of Riemann surfaces with a given topological type. That is, $T(R \backslash\{p\})$ factored by this group is a complex fiber space over $T(R)$ with the property that the fiber over each point $\tau$ in $T(R)$ is a Riemann surface with marked complex structure equal to the complex structure of $R_{\tau}$.

This paper concerns a similar setup but with the following differences. We assume $R$ is an open set in the extended complex plane $\overline{\mathbb{C}}$, possibly disconnected, and that its complement $E=\overline{\mathbb{C}} \backslash R$ contains three or more points. In this setting we define Teichmüller equivalence by isotopy and post composition by Möbius transformations. After normalizing at three points of $E$, the deformations of $R$ in $\overline{\mathbb{C}}$ determine sets $f(E)$ complementary to $f(R)$. Thus we may also think of the points of the Teichmüller space as marked deformations of closed subsets $E$ of $\overline{\mathbb{C}}$. This Teichmüller space is denoted by $T(E)$, so the forgetful map is

$$
\Psi: T(E \cup\{p\}) \rightarrow T(E) .
$$

The fiber $\mathbb{K}=\Psi^{-1}([i d])$ is a one-dimensional marked deformation space that tracks the motion of $p$ in the component $R_{j}$ of $\overline{\mathbb{C}}-E$ where $p$ is located and for deformations in $\mathbb{K}$ the only point of $E \cup\{p\}$ that moves is $p$ itself.

Any infinitesimal form for a metric on $T(E \cup\{p\})$ restricts to an infinitesimal form on $\mathbb{K}$. We focus attention on two special cases, namely, the Teichmüller and Kobayashi infinitesimal metrics, $\operatorname{Kob}(\tau, V)$ and $\operatorname{Teich}(\tau, V)$, which are defined in (6) and (7) in Section 2. These metrics are infima over the families of holomorphic functions from the unit disc into $\mathbb{K}$ and into $T(E \cup\{p\})$ with derivatives mapping to a given tangent direction. Since $\mathbb{K} \subset T(E \cup\{p\})$, the family of such functions with image in $\mathbb{K}$ is smaller and so it turns out that

$$
\operatorname{Teich}(\tau, V) \leq K o b(\tau, V) .
$$

In [8] for Riemann surfaces a reverse inequality is shown, namely,

$$
\operatorname{Kob}(\tau, V) \leq 2 \operatorname{Teich}(\tau, V),
$$

where $\tau$ is a point of $\mathbb{K} \subset T(E \cup\{p\})$ and $V$ is a tangent vector at $\tau$.

The metric densities $K o b(\tau, V)$ and $\operatorname{Teich}(\tau, V)$ on $\mathbb{K}$ induce metric densities on $R$, where the former is the Poincaré metric $\rho(z)|d z|$ on the component of $R$ that contains $p$ and the latter is another metric density $\lambda(z)|d z|$, which we have called the Teichmüller density in [8]. Several authors have studied the comparison between $\rho$ and $\lambda$ (see [3, 8, 10, 12, 13, 16]), but in all of these $p$ is viewed as a variable point on a connected Riemann surface. We give two ways of constructing $\lambda$ on $R=\overline{\mathbb{C}}-E$ and show that the ratio $\lambda / \rho$ is a function defined on $T(R \backslash\{p\})$ bounded above and below by 1 and $1 / 2$. In addition, we show that the ratio is strictly less than 1 unless $R$ has only one component and that component is equal to the Riemann sphere with three points removed. We also show that $\lambda(p) / \rho(p)$ is strictly larger than $1 / 2$ unless $p$ belongs to a simply connected component of $R$.

\section{Teichmüller SPACES}

Let $E$ be a closed subset of the extended complex plane $\overline{\mathbb{C}}$ and consider the family $\mathcal{F}$ of all quasiconformal self-maps $f$ of $\overline{\mathbb{C}}$. Let two elements $f_{0}$ and $f_{1}$ in $\mathcal{F}$ 
be equivalent if there is a Möbius transformation $A$ that carries $f_{0}(E)$ to $f_{1}(E)$ and an isotopy through quasiconformal self-maps $g_{t}, 0 \leq t \leq 1$, of $\overline{\mathbb{C}}$ such that

(i) $g_{0}(z)=A \circ f_{0}(z)$ for all $z$ in $\overline{\mathbb{C}}$,

(ii) $g_{1}(z)=f_{1}(z)$ for all $z$ in $\overline{\mathbb{C}}$, and

(iii) $A \circ f_{0}(p)=g_{t}(p)=f_{1}(p)$ for all $t$ with $0 \leq t \leq 1$ and for all $p$ in $E$.

By definition the Teichmüller space $T(E)$ is the space $\mathcal{F}$ factored by this equivalence relation. If $E$ contains zero, one, two or three points, there are no nontrivial quasiconformal deformations and the Teichmüller space $T(E)$ consists of only one point. To avoid this trivial situation we always assume $E$ contains three or more points, say $z_{1}, z_{2}$ and $z_{3}$. Since the definition of $T(E)$ is independent of post composing quasiconformal self-maps of $\overline{\mathbb{C}}$ by Möbius transformations, we also assume that these quasiconformal maps fix the three points $z_{1}, z_{2}$ and $z_{3}$. This means that in part (iii) of the definition of equivalence of elements of $\mathcal{F}$ the Möbius transformation $A$ is the identity. Since pullback by Möbius transformations induces isomorphisms of Teichmüller spaces, without loss of generality we may also assume that these three points are 0,1 and $\infty$.

The class of the identity map $i d(z)=z$ in $T(E)$ denoted by $[i d]_{E}$ represents a special point which we call the base point. If $g$ is a quasiconformal self-map of $\overline{\mathbb{C}}$, then there is the natural isomorphism $g^{*}$ from $T(g(E))$ to $T(E)$ that carries the class $[f]_{g(E)}$ to the class of $[f \circ g]_{E}$. In particular, it carries the base point of $T(g(E))$ to $[g]_{E}$ in $T(E)$, and the point $\left[g^{-1}\right]_{g(E)}$ in $T(g(E))$ to the base point of $T(E)$.

\section{2. $\mathbb{K}$ AS A HOLOMORPHIC MOTION}

If we take a point $p$ in any component $R_{j}$ of $R=\overline{\mathbb{C}} \backslash E$, then $E \cup\{p\}$ is also a closed set and there is a natural map $\Psi: T(E \cup\{p\}) \rightarrow T(E)$, called the forgetful map, defined by

$$
\Psi\left([f]_{E \cup\{p\}}\right)=[f]_{E} .
$$

It is well defined because quasiconformal maps in an isotopy joining two representatives of $[f]_{E \cup\{p\}}$ fix all of the points of $E \cup\{p\}$. These quasiconformal maps automatically extend continuously to $p$, and the same isotopy is an isotopy of maps that fix the points of $f(E)$.

By a theorem of Bers and Lakic, [2, 11] and [7, page 65], the cotangent space to $T(E)$ for any closed set $E$ in $\overline{\mathbb{C}}$ containing the point $\infty$ is equal to the $L_{1}$-closure of the rational meromorphic functions on $\overline{\mathbb{C}}$ that are holomorphic on $\mathbb{C}-E$ and have at most simple poles on $E$. We shall denote this space by $A(E)$; it is the space of integrable holomorphic quadratic differentials on $\overline{\mathbb{C}}-E$. The derivative of $\Psi$ at any point $\tau=[f]_{E \cup\{p\}}$ in $T(E \cup\{p\})$ is the linear map that is dual to the inclusion $A(f(E)) \subset A(f(E) \cup\{f(p)\})$. It follows from Theorem 3, which we prove later, that there always is a quadratic differential in $A(f(E) \cup\{f(p)\})$ and not in $A(f(E))$, so this derivative is surjective at every point of $T(E \cup\{p\})$. If furthermore $[f] \in \mathbb{K}$, then $f$ is an orientation-preserving map that fixes the boundary of $R_{j}$, and so $f(p)$ belongs to $R_{j}$. If $g$ is any map in the equivalence class of $[f]_{E \cup\{p\}}$ in $T(E \cup\{p\})$, then by part (iii) in the definition of equivalence for $T(E \cup\{p\}), g(p)=f(p)$. Therefore, there is an evaluation map $\tilde{F}$ from $T(E \cup\{p\})$ into $\overline{\mathbb{C}}$ defined by

$$
\tilde{F}\left([f]_{E \cup\{p\}}\right)=f(p) .
$$


If we denote by $F$ the restriction of $\tilde{F}$ to $\mathbb{K}$, then $F$ maps $\mathbb{K}$ to $R_{j}$. Moreover, because $R_{j}$ is open and connected one can find an isotopy of quasiconformal maps $f_{t}$ that equals the identity outside of $R_{j}$ and carries $p$ to a point $f_{t}(p)=p_{t}$ in $R_{j}$ such that $p_{1}=f_{1}(p)$ is an arbitrary point of $R_{j}$. The equivalence class $\left[f_{t}\right]_{E \cup\{p\}}$ is a curve in $\mathbb{K}$ connecting $[i d]$ to $\left[f_{1}\right]_{E \cup\{p\}}$ and $F\left(\left[f_{1}\right]_{E \cup\{p\}}\right)=p_{1}$. This shows that $F$ maps $\mathbb{K}$ onto $R_{j}$.

Let $\Pi$ be a universal covering from the unit disc $\Delta$ onto $R_{j}$ such that $\Pi(0)=p$. The map $F$ is also a covering mapping onto $R_{j}$ in the sense that for any $q$ in $R_{j}$ there is a disc $D$ in $R_{j}$ containing $q$ such that $F^{-1}(D)$ is a disjoint union of conformal discs $D_{j}$ in $\mathbb{K}$ such that $F$ restricted to $D_{j}$ is a homeomorphism of $D_{j}$ onto $D$. Since $\Pi$ is a universal covering, there is a unique lift $\tilde{\Pi}$ mapping from $\Delta$ to $\mathbb{K}$ such that $F \circ \tilde{\Pi}=\Pi$ and $\tilde{\Pi}(0)$ is the base point in $T(E \cup\{p\})$.

Theorem 1. The lift $\tilde{\Pi}$ of $\Pi$ is bijective and conformal from $\Delta$ onto $\mathbb{K}$.

Proof. We have already observed that $F$ maps $\mathbb{K}$ onto $R_{j}$ and that it is a surjective covering of $R_{j}$ and so $F$ lifts to a covering $\tilde{\Pi}$ from $\Delta$ onto $\mathbb{K}$. If we can show $\mathbb{K}$ is simply connected, then $\tilde{\Pi}$ must be an isomorphism. By a theorem of Lieb (see [5]) the Teichmüller space $T(E)$ is isomorphic to

$$
\left(\Pi_{k} T\left(R_{k}\right)\right) \times M(E),
$$

where $R_{k}$ runs over all the components of the complement of $E$ in $\overline{\mathbb{C}}$ and $M(E)$ is the open ball of measurable complex-valued functions $\mu$ supported on $E$ with $\|\mu\|_{\infty}<1$. The isomorphism carries the Beltrami coefficients representing the points in each of the Teichmüller spaces $T\left(R_{j}\right)$ to Beltrami coefficients defined independently on the domains $R_{j}$. The Teichmüller metric is induced by the $L_{\infty}$ metric on Beltrami coefficients. Now assume $p$ is in a particular component $R_{j}$ of the complement of $E$. By Bers' theorem [3] the fiber over the identity of the forgetful map from $T\left(R_{j}-\{p\}\right)$ to $T\left(R_{j}\right)$ is simply connected, and by Lieb's theorem it is isomorphic to the fiber over the identity of

$$
\Psi:\left(\Pi_{k \neq j} T\left(R_{k}\right)\right) \times M(E) \times T\left(R_{j}-\{p\}\right) \rightarrow\left(\Pi_{k \neq j} T\left(R_{k}\right)\right) \times M(E) \times T\left(R_{j}\right) .
$$

So the points of the fiber over the identity in this projection are represented precisely by the points of the fiber over the identity of $T\left(R_{j}-\{p\}\right) \rightarrow T\left(R_{j}\right)$.

For the next result we first give some basic facts about the Kobayashi metric on a complex manifold $M$. Its infinitesimal form $\operatorname{Kob}_{M}(\tau, V)$ is a positive number assigned to each tangent vector $V$ at a point $\tau$ in $M$ by the following extremal problem:

$$
K_{o b}(\tau, V)=\inf \left\{|k| \mid k g^{\prime}(0)=V\right\},
$$

where the infimum is taken over all nonnegative numbers $k$ and all holomorphic functions $g$ mapping the unit disc $\Delta$ into $M$.

Because it is known that for any Teichmüller space $T, \operatorname{Kob}_{T}(\tau, V)=\operatorname{Teich}_{T}(\tau, V)$, [6] and [14, the infinitesimal norm $\operatorname{Teich}_{T}(\tau, V)$ is given by

$$
K_{o b}(\tau, V)=\inf \left\{|k| \mid k g^{\prime}(0)=V\right\}=\operatorname{Teich}_{T}(\tau, V)=\inf \left\{\|\bar{\partial} \tilde{V}\|_{\infty}\right\},
$$

where the first infimum is taken over all $k$ and all holomorphic functions $g$ mapping $\Delta$ into $T$ with $g(0)=\tau$ and the second infimum is over all continuous vector fields $\tilde{V}$ whose restriction to $f^{\mu}(E \cup\{p\})$ is equal to $V$ and where $f^{\mu}$ represents the point $\tau \in T(E \cup\{p\})$. However, for complex submanifolds $M$ of $T$, Kobayashi's 


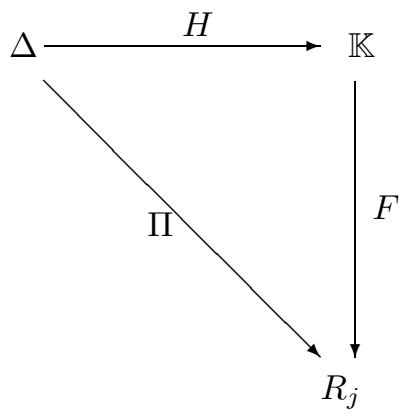

FIGURE 1

infinitesimal form for $M$ may differ from Teichmüller's infinitesimal form for $T$ restricted to $M$ since the extremal problem defining $\operatorname{Kob}_{M}(\tau, V)$ involves the further requirement that the holomorphic function $g$ must have image contained in $M$. Thus, obviously,

$$
\operatorname{Teich}_{M}(\tau, V) \leq \operatorname{Kob}_{M}(\tau, V) .
$$

We will see that this is usually a strict inequality when $M=\mathbb{K}$.

Schwarz's lemma implies that Kobayashi's metric for a complex disc coincides with Poincaré's metric. This observation is expressed in the following proposition.

Proposition 1. The Kobayashi density $K_{0} b_{\mathbb{K}}(\tau, V)$ on $\mathbb{K}$ and the Poincaré density $\rho$ on $R_{j}$ are related by the following formula:

$$
\rho(F([f]))|d F(V)|=K o b_{\mathbb{K}}([f], V) .
$$

Proof. Since $\Pi$ is a universal covering of $\Delta$ onto $R_{j}$ mapping 0 to $p, \frac{1}{\left|\Pi^{\prime}(0)\right|}$ realizes the smallest value of $\frac{1}{\left|g^{\prime}(0)\right|}$, where $g(0)=p$ and $g$ is holomorphic mapping $\Delta$ into $R_{j}$.

The next theorem identifies the unique lifting $\tilde{\Pi}$ of the previous theorem in a different way. Define a normalized holomorphic motion $h(t, z)$ of $E \cup\{p\}$ by the following formula:

$$
h(t, z)=\left\{\begin{array}{lll}
z & \text { for } \quad z \in E \\
\Pi(t) & \text { for } \quad z=p
\end{array}\right.
$$

By Slodkowski's 15 extension theorem, $h$ extends to a normalized holomorphic motion $\tilde{h}$ of the extended complex plane. The map $H$ that sends $t$ to the class of $z \mapsto \tilde{h}(t, z)$ is a holomorphic map from the unit disc into $T(E \cup\{p\})$. Furthermore, since $\tilde{h}(t, z)=h(t, z)=z$ for all $t$ and all $z$ in $E$, the curve $H(t)$ represents the homotopy class that keeps all $H(t)$ in the same class in $T(E)$. Since $H(0)$ is the identity map, the image of $H$ is in $\mathbb{K}$. Since $F(H(t))=\tilde{h}(t, p)=h(t, p)=\Pi(t)$, the diagram in Figure 1 commutes.

Let $\gamma$ be any path in $\overline{\mathbb{C}} \backslash E$ such that $\gamma(0)=p$. Take a lift $\tilde{\gamma}$ of $\gamma$ under the universal covering map $\Pi$ and with the property $\tilde{\gamma}(0)=0$. Then the path $\hat{\gamma}=H(\tilde{\gamma})$ is a path in $\mathbb{K}$ that starts at the base point and whose image under $F$ is $\gamma$. Since from the previous theorem $\mathbb{K}$ is simply connected, $H$ is a biholomorphic surjection. Therefore, we have proved the following theorem. 
Theorem 2. The map $F$ is a holomorphic covering from $\mathbb{K}$ onto $R_{j}$. Furthermore, the holomorphic motion $h$ in (10) defines the whole fiber $\mathbb{K}$. More precisely, for every $\tau$ in $\mathbb{K}$, there exists $t$ in $\Delta$ such that $\tau=[z \mapsto \tilde{h}(t, z)]$.

\section{COMPARING METRICS ON $\mathbb{K}$}

We now define the infinitesimal form of a metric $\lambda$ naturally associated to any hyperbolic Riemann surface which turns out to be different from but equivalent to the Poincaré metric $\rho$. In [8] we have called it the Teichmüller density because it is the metric on $R$ induced by $T e i c h(\tau, V)$ on the fiber $\mathbb{K}=\Psi^{-1}([i d])$. It will turn out that the ratio $\lambda(p) / \rho(p)$ is a real-valued functional on domains $E \cup\{p\}$ that takes values always between $1 / 2$ and 1 , and takes the extreme value $1 / 2$ only when $p$ is in a simply connected component of $R$ and the extreme value 1 only when $R$ is the complement of three points in the Riemann sphere.

As a starting point we make the following definitions.

Definition 1. The infinitesimal Teichmüller norm of a vector field $V$ defined on $E \cup\{p\}$ is

$$
\|V\|_{T}=\inf \left\{\|\bar{\partial} \tilde{V}\|_{\infty}\right\}
$$

where the infimum is taken over all continuous, complex-valued functions $\tilde{V}$ with distributional derivatives and $\tilde{V}$ equal to $V$ on $E \cup\{p\}$.

Definition 2. The Teichmüller density $\lambda(p)$ evaluated at a point $p$ in $R_{j}$ is equal to $\left\|V_{0}\right\|_{T}$, where $V_{0}$ is the function defined on $E \cup\{p\}$ which is equal to zero on $E$ and equal to 1 at $p$.

Proposition 2. The Teichmüller density Teich $\mathbb{K}([f], V)$ on $\mathbb{K}$ is related to the Teichmüller density $\lambda$ on $R_{j}$ by the formula:

$$
\lambda(F([f]))|d F(V)|=\operatorname{Teich}_{\mathbb{K}}([f], V),
$$

where $F: \mathbb{K} \rightarrow R_{j}$ is the evaluation map $F([f])=f(p)$.

Proof. We first prove this equality for $[f]=[i d]$. If $[f]=[i d]$, then $F([f])=p$. If $V$ is the zero vector, then $d F(V)=0$ and (12) reduces to $0=0$. Suppose $V$ is a nonzero vector field tangent to $\mathbb{K}$. Then $V$ vanishes on $E, V(p) \neq 0$ and $\lambda(p)=\|V / V(p)\|_{T}$. Also $\left.d F\right|_{[i d]}(V)=V(p)$, which follows from [1], page 104. So the left-hand side of (12) is equal to $\|V\|_{T}$. From Definition 1 and (7) this is equal to the right-hand side of (12).

Let $[f]$ be any point in $\mathbb{K}$ and $G:$ Teich $(E \cup f(p)) \rightarrow$ Teich $(E \cup p)$ given by $G([g])=[g \circ f]$ is an isomorphism. Also, if $\Psi: T(E \cup\{p\}) \rightarrow T(E)$ and $\Psi_{G}:$ $T(E \cup\{f(p)\}) \rightarrow T(E)$ are the forgetful maps, then $\Psi \circ G=\Psi_{G}$. If $\mathbb{K}^{\prime}$ is the fiber over the identity of $\Psi_{G}$ and $\mathbb{K}$ is the fiber over the identity of $\Psi$, then $G$ restricted to $\mathbb{K}^{\prime}$ is an isomorphism of $\mathbb{K}^{\prime}$ onto $\mathbb{K}$. For $[g] \in \mathbb{K}^{\prime},(F \circ G)([g])=F([g \circ f])=g(f(p))$. Thus $F \circ G$ is the evaluation map for $g$ at the point $f(p)$. By what we have just proved,

$$
\left.\lambda(F G([i d])) d(F G)\right|_{[i d]}\left(\left(G^{-1}\right)^{*} V\right)=\operatorname{Teich}_{\mathbb{K}^{\prime}}\left([i d],\left(G^{-1}\right)^{*} V\right) .
$$

But $\lambda(F \circ G([i d]))=\lambda(f(p))$. Also,

$$
\operatorname{Teich}_{\mathbb{K}^{\prime}}\left([i d],\left(G^{-1}\right)^{*} V\right)=\operatorname{Teich}_{\mathbb{K}}\left([g], d G_{[i d]}\left(G^{-1}\right)^{*}(V)\right)=\operatorname{Teich}_{\mathbb{K}}([g], V) .
$$


The chain rule implies

$$
\left.d(F G)\right|_{i d}\left(\left(G^{-1}\right)^{*} V\right)=d F_{[f]}\left(d G_{[i d]}\left(G^{-1}\right)^{*}(V)\right)=d F_{[f]}(V),
$$

and so (12) follows from (13).

To get a lower bound for the infinitesimal form $\lambda(p)$ we construct a quadratic differential $\psi_{p}$ canonically associated with any Riemann surface $R$ and a point $p$ in $R$. Let $\Pi$ be a universal covering of a component $R$ of the complement of $E$ in $\overline{\mathbb{C}}$. Assume $\Pi$ is normalized so that $\Pi(0)=p$ and $\Pi^{\prime}(0)$ is positive. Let $\Gamma$ be the universal covering group for $\Pi$. $\Gamma$ is a fixed point free discrete group of Möbius transformations acting on the unit disc $\Delta, \Pi \circ \gamma=\Pi$ for every $\gamma \in \Gamma$, and the factor space $\Delta / \Gamma$ is conformal to $R$.

Theorem 3. The Poincaré theta series of $\psi(z)=\frac{1}{\pi z}$,

$$
\sum_{\gamma \in \Gamma} \psi(\gamma(z)) \gamma^{\prime}(z)^{2}=\sum_{\gamma \in \Gamma} \frac{\gamma^{\prime}(z)^{2}}{\pi \gamma(z)}=\psi_{p}(z),
$$

defines a quadratic differential $\psi_{p}$ on $R$ which is holomorphic on $R-\{p\}$, which has a simple pole at $p$ and for which

$$
1 \leq\left\|\psi_{p}\right\| \leq 2 .
$$

Proof. The tangent vector $V=H^{\prime}(0)$ to the holomorphic motion $H(t, z)$ from Section 2 vanishes on $E$ and satisfies $V(p)=\Pi^{\prime}(0)$. Put

$$
V_{0}=\frac{V}{\Pi^{\prime}(0)} \text {. }
$$

Since $1 /\left|\Pi^{\prime}(0)\right|=\rho(p)$ and since by Schwarz's lemma $\|V\|_{T} \leq 1$, we obtain

$$
\left\|V_{0}\right\|_{T}=\rho(p)\|V\|_{T} \leq \rho(p) .
$$

On the other hand,

$$
\left\|V_{0}\right\|_{T} \geq \frac{\left|V_{0}(\hat{\varphi})\right|}{\|\hat{\varphi}\|}
$$

for every integrable quadratic differential $\hat{\varphi}$ holomorphic on $R-\{p\}$. In particular

$$
\rho(p) \geq\left\|V_{0}\right\|_{T} \geq \frac{\left|V_{0}\left(\psi_{p}\right)\right|}{\left\|\psi_{p}\right\|}
$$

for the quadratic differential $\psi_{p}$ given by the Poincaré theta series (14).

By the Ahlfors-Bers density theorem, $\psi_{p}$ is the $L_{1}$-limit of integrable holomorphic functions $\psi_{p}$ in the plane with a finite number of simple poles located at $p$ and on the boundary of $R_{j}$. Thus to evaluate $V\left(\psi_{p}\right)$ we may assume it has only a finite number of poles. But if we let $\tilde{V}_{0}$ be a continuous extension of $V_{0}$ with bounded $\bar{\partial}$-derivative, then

$$
\begin{gathered}
V_{0}\left(\psi_{p}\right)=\iint \bar{\partial} \tilde{V}_{0} \psi_{p} d x d y=\iint \bar{\partial} \tilde{V}_{0} \psi_{p} \frac{d \bar{z} d z}{2 i} \\
=\iint d\left(\tilde{V}_{0} \psi_{p} \frac{d z}{2 i}\right)=-\pi r e s\left(\psi_{p}, p\right) .
\end{gathered}
$$


The only residue of $\left(\tilde{V}_{0} \psi_{p} d z\right)$ is at $p$ and this residue is equal to $1 /\left(\pi \Pi^{\prime}(0)\right)$, where $\Pi$ is a universal covering of $R_{j}$ with $\Pi(0)=p$. Since $\rho(p)=1 /\left|\Pi^{\prime}(0)\right|$,

$$
\left|V_{0}\left(\psi_{p}\right)\right|=\rho(p)
$$

and from (17) we get

$$
\rho(p) \geq\left\|V_{0}\right\|_{T} \geq \frac{\rho(p)}{\left\|\psi_{p}\right\|}
$$

which yields $\left\|\psi_{p}\right\| \geq 1$.

On the other hand,

$$
\left\|\psi_{p}\right\|=\left|\iint_{\omega} \sum \frac{\gamma^{\prime}(z)^{2}}{\pi \gamma(z)}\right| \leq \iint_{\omega} \sum\left|\frac{\gamma^{\prime}(z)^{2}}{\pi \gamma(z)}\right|=\iint_{|z|<1} \frac{1}{|\pi z|} d x d y=2,
$$

where $\omega$ is a fundamental domain in $\Delta$ for the covering group.

Theorem 4 ([8]). The infinitesimal form $\lambda$ on each domain $R_{j}$ is equivalent to the Poincaré infinitesimal form $\rho$ on that domain. More precisely,

$$
(1 / 2) \rho(p) \leq \lambda(p) \leq \rho(p) .
$$

Proof. The right-hand side of (20) follows from (16). The left-hand side follows by choosing the specific quadratic differential $\psi_{p}$ on $R_{j} \backslash\{p\}$ given in Theorem 3 . The theta series of $\psi(z)=1 /(\pi z)$ is summed over all covering transformations $\gamma$ for the covering map $\Pi$ of $R_{j}$. Since $\left\|\psi_{p}\right\| \leq 2$, the definition of $\lambda(p)$ yields

$$
\lambda(p) \geq \frac{\left|V_{0}\left(\psi_{p}\right)\right|}{\left\|\psi_{p}\right\|}=\frac{\rho(p)}{\left\|\psi_{p}\right\|} \geq \frac{\rho(p)}{2} .
$$

Just as with the Poincaré metric, the integrated form of $\lambda(p)|d p|$ becomes a metric by the following recipe:

$$
\lambda\left(p_{1}, p_{2}\right)=\inf \int_{\gamma} \lambda(\gamma(t))\left|\gamma^{\prime}(t) d t\right|,
$$

where the infimum is over all continuous, piecewise differentiable curves $\gamma$ in $R$ such that $\gamma(0)=p_{1}$ and $\gamma(1)=p_{2}$.

Corollary 1. The metrics $\lambda\left(p_{1}, p_{2}\right)$ and $\rho\left(p_{1}, p_{2}\right)$, which are the integrated forms of $\lambda(p)|d p|$ and $\rho(p)|d p|$, satisfy the following inequality:

$$
(1 / 2) \rho\left(p_{1}, p_{2}\right) \leq \lambda\left(p_{1}, p_{2}\right) \leq \rho\left(p_{1}, p_{2}\right) .
$$

Proof. To define the integral in (22) it is helpful to know that the integrand is continuous, which is justified by Corollary 2 given in the next section. The inequality of this corollary then follows directly from the corresponding inequality for the infinitesimal metrics.

\section{Residues as Densities}

Theorem 3 shows the existence of a special quadratic differential with a simple pole at any given point $p$ in $R$. We now show that the residue of a meromorphic $n$-differential is an $(n-1)$-differential and, consequently, the residue of a quadratic differential is a one-form. We state this observation as a lemma. 
Lemma 1. Suppose $\varphi=\varphi(z)(d z)^{n}$ is a meromorphic $n$-differential in a neighborhood of $p$. Then the residue of $\varphi$ at $p$ is an $(n-1)$-differential.

Proof. Let $w$ and $z$ be charts such that $z=0$ in $U$ and $w=0$ in $V$ correspond to the point $p$. Let $\varphi_{U}(z)$ and $\varphi_{V}(w)$ be the expressions for $\varphi$ in terms of the parameters $z$ and $w$ so that

$$
\varphi_{U}(z)=\varphi_{V}(f(z)) f^{\prime}(z)^{n}
$$

where $w=f(z)$ is the transition mapping. Then

$$
\operatorname{res}\left(\varphi_{V}, p\right) f^{\prime}(0)^{n-1}=\frac{1}{2 \pi i} \int_{w(\gamma)} \varphi_{V}(w) f^{\prime}(0)^{n-1} d w=\frac{1}{2 \pi i} \int_{\gamma} \varphi_{U}(z) \frac{f^{\prime}(0)^{n-1}}{f^{\prime}(z)^{n-1}} d z .
$$

Here $\gamma$ is a simple closed curve in the $z$-plane winding once around $z=0$ and oriented in the counterclockwise direction and $w(\gamma)$ has the same properties with respect to $w=0$ in the $w$-plane. The integrals depend only on the homotopy class provided $\gamma$ lies in a sufficiently small neighborhood of 0 . Since $f$ is univalent, the fraction $f^{\prime}(0)^{n-1} / f^{\prime}(z)^{n-1}$ becomes uniformly close to 1 as that neighborhood becomes sufficiently small. Therefore, the integral on the right-hand side is equal to

$$
\frac{1}{2 \pi i} \int_{\gamma} \varphi_{U}(z) d z=\operatorname{res}\left(\varphi_{U}, p\right)
$$

In the case that $\varphi$ is a quadratic differential, $\operatorname{res}(\varphi, p) d p$ is a holomorphic oneform and, if $\varphi$ has at most simple poles, then $\operatorname{res}(\varphi, p)=0$ if and only if $\varphi$ extends holomorphically at $p$. Moreover, the absolute value $|r e s(\varphi, p) d p|$ determines a density, that is, an infinitesimal metric. In addition to its residue, any integrable quadratic differential $\varphi$ holomorphic on $E \cup\{p\}$ carries another invariant, namely, its $L^{1}$ norm. Thus we can ask for the largest residue of an integrable quadratic differential holomorphic on $R \backslash\{p\}$ subject to the condition that its $L^{1}$ norm is equal to one. Obviously, a differential that realizes this largest value will be identically equal to zero on every component of $R$ that does not contain $p$.

Putting these pieces together we obtain the following theorem, which serves as an alternative definition of the Teichmüller density $\lambda$.

\section{Theorem 5.}

$$
\lambda(p)=\pi \sup _{\|\varphi\|=1} \operatorname{Re} \operatorname{res}(\varphi, p)
$$

where the supremum is over all quadratic differentials holomorphic in $R \backslash\{p\}$ with $L^{1}$ norm one. Moreover, there is a unique quadratic differential $\varphi_{p}$ that realizes the supremum in (23).

Proof. Let $V(p)=1$ and $V$ vanish on $E$, and let $A(E \cup\{p\})$ be the Banach space of integrable quadratic differentials $\varphi$ holomorphic on the complement of $E \cup\{p\}$ with norm given by

$$
\|\varphi\|=\int_{R}|\varphi| d x d y .
$$

By the Hahn-Banach and Riesz representation theorems, there exists a continuous extension $\tilde{V}$ of $V$ with bounded $\bar{\partial}$-derivative such that

$$
\|\bar{\partial} \tilde{V}\|_{\infty}=\sup \left\{\left|\iint_{R} \bar{\partial} \tilde{V} \varphi\right|\right\},
$$


where the supremum is over all $\varphi \in A(R \backslash\{p\})$ with $\|\varphi\|=1$, and where $\tilde{V}$ is a continuous extension of $V$ to $R$.

By the same residue calculation given in the proof of Theorem 3 we find that

$$
\iint_{R} \bar{\partial} \tilde{V} \varphi=-\pi r e s(\varphi, p)
$$

Therefore the infimum in Definition 1 is equal to the supremum in (23).

To prove the second part of the theorem, the evaluation of the residue of $\varphi$ at a point $p$ is a linear functional $V \in A(E \cup\{p\})^{*}$ given by the formula

$$
V(\varphi)=\frac{1}{2 \pi i} \int_{\gamma} \varphi(z) d z
$$

where $\gamma$ is a closed curve with winding number 1 around the point $p$. Now suppose $\varphi_{n}$ is a sequence of differentials in $A(E \cup\{p\})$ with $\left\|\varphi_{n}\right\|=1$ such that $\operatorname{Re} \pi \operatorname{res}\left(\varphi_{n}, p\right)$ approaches the supremum in (23). From equicontinuity of bounded holomorphic functions there must be a subsequence of $\varphi_{n}$ which converges uniformly on compact subsets of $R-\{p\}$. But it is not possible for any subsequence of $\varphi_{n}$ to converge uniformly on compact subsets to any quadratic differential $\varphi$ with $\|\varphi\|<1$, because replacing $\varphi$ by $\varphi /\|\varphi\|$ gives a quadratic differential of norm 1 with a larger residue. Therefore, a subsequence of $\varphi_{n}$ converges uniformly on compact subsets to $\varphi$ and $\left\|\varphi_{n}\right\|=1=\|\varphi\|$, which implies $\left\|\varphi_{n}-\varphi\right\|$ converges to zero and so $\varphi$ realizes the supremum in (23). The point $\varphi$ on the unit sphere realizing the supremum is unique because the unit ball of $A(E \cup\{p\})$ is strictly convex. Thus, every convergent subsequence of $\varphi_{n}$ converges to the same limit, which implies the original sequence itself converges to that limit.

Corollary 2. $\lambda(p)$ is continuous in $p$.

Proof. Assume $|q-p|<\delta$. Then the Bers' $\mathcal{L}$-operator [7] yields a Banach space isomorphism $\mathcal{L}$ from $A(E \cup\{q\})$ onto $A(E \cup\{p\})$ such that

$$
\max \left\{\|\mathcal{L}\|,\left\|\mathcal{L}^{-1}\right\|\right\}<1+\epsilon(\delta),
$$

where $\epsilon(\delta)$ is bounded by the $L_{\infty}$-norm of the Beltrami coefficient of any quasiconformal self-map of $R$ that carries $q$ to $p$ and is equal to the identity outside a fixed disc of radius $\delta_{1}$ with center $p$ and with $|q-p|<(1 / 2) \delta_{1}$.

Theorem 6. Let $\rho$ be the Poincaré metric for $R$. That is, in any component $R_{j}$ of $R, \rho$ is equal to $\rho_{R_{j}}$, the Poincaré metric for the component $R_{j}$. Then for every quadratic differential $\varphi$ in $A(R \backslash\{p\})$, we have

$$
|\operatorname{res}(\varphi, p)| \leq \frac{\rho(p)}{\pi}\|\varphi\|
$$

Proof. From Theorem 5

$$
\lambda(p) \geq \pi|\operatorname{res}(\varphi, p)| /\|\varphi\|
$$

and we already know $\rho(p) \geq \lambda(p)$, so the theorem follows. 


\section{Domain differentials}

In the previous two sections we constructed two quadratic differentials $\psi_{p}$ and $\varphi_{p}$ in $A(E \cup\{p\})$ canonically associated to a plane domain and a point $p$ in that domain. $\psi_{p}$ is an element of $A(E \cup\{p\})$ given by a theta series of the function $q(z)=1 /(\pi z)$ summed over the elements of a Fuchsian covering group acting on the unit disc and chosen so that 0 covers $p$ and $\Pi^{\prime}(0)$ is positive. $\varphi_{p}$ is an element of $A(E \cup\{p\})$ that maximizes the real part of $V_{0}(\varphi)$ with $\|\varphi\|=1$, where $V_{0}$ is a vector field vanishing on $E$ and equal to 1 at $p$. We have already seen in Theorem [5] that $\varphi_{p}$ realizing this extreme is uniquely determined. These two differentials determine the Teichmüller and Poincaré densities by the following formulae:

$$
\lambda(p)=\pi \operatorname{Re} \operatorname{res}\left(\varphi_{p}, p\right) \text { and } \rho(p)=\pi \operatorname{Re} \operatorname{res}\left(\psi_{p}, p\right) .
$$

The left-hand formula comes from Theorem 5 and the right-hand comes from (18) and (19).

Before stating the next theorem we need to recall the definition of the Zygmund Banach space $Z(E)$ on an arbitrary closed set $E \subset \overline{\mathbb{C}}$. By definition, $Z(E)$ consists of all continuous complex-valued vector fields $V$ defined on $E$ with the property that $V$ has a continuous extension $\tilde{V}$ to the complex plane $\overline{\mathbb{C}}$ such that $\|\bar{\partial} \tilde{V}\|_{\infty}<\infty$. The assumption that the vector field $V(z) \frac{\partial}{\partial \bar{z}}$ is continuous at $z=\infty$ implies there is a constant $C$ such that $|\tilde{V}(z)| \leq C|z|^{2}$. Definition 1 defines the Teichmüller norm of $V$, and one easily shows that $\|V\|_{T}=0$ if and only if $V$ is the restriction of a quadratic polynomial to $E$.

There is another natural norm on $Z(E)$, which we call the cross ratio norm, and it is given by a supremum of cross ratios. To define the cross ratio norm, assume $Q=\{a, b, c, d\}$ is an arbitrary quadruple of four points contained in $E$ and form

$$
\|V\|_{c r}=\sup \left|\operatorname{cr}(Q) \rho_{01}(\operatorname{cr}(Q)) V[Q]\right| \text {, }
$$

where

$$
V[Q]=\frac{V(b)-V(a)}{b-a}-\frac{V(c)-V(b)}{c-b}+\frac{V(d)-V(c)}{d-c}-\frac{V(a)-V(d)}{a-d}
$$

and where $\rho_{01}(p)$ is the Poincaré metric of the domain $\overline{\mathbb{C}}-\{0,1, \infty\}$. In $[7$, page $67]$ it is shown that the two norms $\|V\|_{T}$ and $\|V\|_{c r}$ are equivalent by universal constants independent of the set $E$. Moreover, $Z(E)$ is a Banach space and the pairing between $A(E)$ and $Z(E)$,

$$
(\varphi, V)=\operatorname{Re} \iint \varphi \bar{\partial} \tilde{V} d x d y
$$

induces an isomorphism from $Z(E)$ onto $A(E)^{*}$.

In general the one-dimensional spaces in $A(E \cup\{p\})$ spanned by $\varphi_{p}$ and $\psi_{p}$ are not equal except, of course, when $A(E \cup\{p\})$ is one dimensional. The only case when $A(E \cup\{p\})$ is one dimensional occurs when $E$ is the smallest possible set, namely, when $E=\{0,1, \infty\}$. These comments lead to the following theorem.

Theorem 7. If $E=\{0,1, \infty\}$, then $\lambda(p)=\rho(p)$. Furthermore inequality (15) in Theorem 3 becomes $\left\|\psi_{p}\right\|=1, \varphi_{p}=\psi_{p}$ and

$$
\psi_{p}(w)=\left(\frac{1}{\pi \Pi^{\prime}(0)}\right) \cdot \frac{p(p-1)}{w(w-1)(w-p)},
$$

where $\Pi$ is the covering map described in Theorem 3 . 
Proof. For every Teichmüller space $T(E \cup\{p\})$, we know that $\left\|\varphi_{p}\right\|=1$ and $1 \leq$ $\left\|\psi_{p}\right\| \leq 2$. When $E=\{0,1, \infty\}, \mathbb{K}=T(E \cup\{p\})$ and so Teichmüller's metric equals Kobayashi's metric because the Teichmüller and Kobayashi metrics coincide on any Teichmüller space. Thus, from Propositions 1 and 2, $\lambda(p)=\rho(p)$. Because of (26) this means $\operatorname{res}\left(\varphi_{p}, p\right)=\operatorname{res}\left(\psi_{p}, p\right)$. Note that $A(\{0,1, p, \infty\})$ is one dimensional and generated by

$$
\frac{p(p-1)}{w(w-1)(w-p)}
$$

which has residue at $p$ equal to 1 . From the calculation preceding (19),

$$
\operatorname{res}\left(\psi_{p}(w), p\right)=\operatorname{res}\left(\frac{1}{\pi z}, 0\right) \cdot\left(\left.\frac{d z}{d w}\right|_{w=p}\right)=\frac{1}{\pi \Pi^{\prime}(0)},
$$

where $w=\Pi(z)$. Thus, after multiplication by $1 /\left(\pi \Pi^{\prime}(0)\right)$, the formula in (27) has the same residue as $\psi_{p}$ at $p$ and so must coincide with $\psi_{p}$. Since both $\varphi_{p}$ and $\psi_{p}$ have the same residue at $p$ and are in the same one-dimensional space, $\varphi_{p}=\psi_{p}$.

Corollary 3 (Agard's formula). The Poincaré metric for $\overline{\mathbb{C}}-\{0,1, \infty\}$ is

$$
\rho(p)=\left(\frac{1}{\pi} \iint\left|\frac{p(p-1)}{w(w-1)(w-p)}\right| d u d v\right)^{-1} .
$$

Proof. From its definition, $\varphi_{p}$ has norm equal to 1 . Since $\psi_{p}=\varphi_{p}, \psi_{p}$ also has norm equal to 1 and (28) is equivalent to the equation $\left\|\psi_{p}\right\|=1$.

Theorem 8. For $p$ in any simply connected component $R_{j}$ of $\overline{\mathbb{C}}-E, \lambda(p)=$ $(1 / 2) \rho(p)$. Moreover, the pullbacks of the quadratic differentials $\varphi_{p}$ and $\psi_{p}$ to the unit disc by the Riemann map that maps $p$ to 0 and has positive derivative at $p$ are equal to $1 /(2 \pi z)$ and $1 /(\pi z)$, respectively.

Proof. Since both metrics are invariant under conformal change of coordinates, to show $\lambda(p)=(1 / 2) \rho(p)$ we only need to show that $\lambda_{\Delta}(0)=\rho_{\Delta}(0) / 2$, where $\Delta$ is the unit disc. Since the covering group is trivial in this case, from Theorem 3 it is obvious that $\psi_{0}(z)=1 /(\pi z)$. We claim that $\varphi_{0}(z)=1 /(2 \pi z)$. Since $\left\|\varphi_{0}(z)\right\|=1$, we know from Theorem 5 that $\lambda_{\Delta}(0) \geq 1 / 2$. The reverse inequality follows by considering the vector field $V(z)=1-|z|$ for $|z| \leq 1$ and $V(z)=0$ for $|z| \geq 1$. Then $V(0)=1$ and $\bar{\partial} V(z)=-(1 / 2) z^{1 / 2} \bar{z}^{-1 / 2}$, so $\|\bar{\partial} V\|_{\infty}=1 / 2$. This shows that any $\varphi$ for which $\operatorname{\pi res}(\varphi, 0)>1 / 2$ must have norm greater than 1 . But from Theorem 5 the quadratic differential with positive residue at 0 that realizes the maximum value of $V(\varphi)$ is unique. Therefore, $\varphi_{0}=1 /(2 \pi z)$.

\section{Kobayashi and Teichmüller eXtremal domains}

In this section we show that the inequalities in (20) in Theorem 4 are sharp except in two special cases. That is, we show $\lambda(p)=\rho(p)$ if and only if $E$ consists of three points and $\lambda(p)=(1 / 2) \rho$ if and only if $p$ is in a simply connected component $R_{j}$ of $R$.

First, we make a general remark about notation. If we are given a continuous infinitesimal metric $\alpha(p)|d p|$ defined on a domain and a differentiable curve $\gamma(t), 0 \leq$ $t \leq 1$, in that domain, we shall denote the $\alpha$-arclength of $\gamma$ by $\alpha(\gamma)$, that is,

$$
\alpha(\gamma)=\int_{0}^{1} \alpha(\gamma(t))\left|\gamma^{\prime}(t)\right||d t|
$$


Just as in formula (22), we define the $\alpha$-distance from $p$ to $q$ by

$$
\alpha(p, q)=\inf \{\alpha(\gamma)\},
$$

where the infimum is taken over all curves $\gamma$ in the domain that join $p$ to $q$.

Theorem 9. If E has exactly three points, then the map $H$ in Figure 1 is an isometry from the unit disc $\Delta$ with the hyperbolic metric onto $\mathbb{K}$ with the infinitesimal form of Teichmüller's metric. That is,

$$
\operatorname{Teich}_{\mathbb{K}}(H(t), H(s))=\rho_{\Delta}(t, s) .
$$

If $E$ is connected, or equivalently, if every component of $R$ is simply connected, then

$$
\operatorname{Teich}_{\mathbb{K}}(H(t), H(s))=\frac{1}{2} \rho_{\Delta}(t, s)
$$

for all $t$ and $s$ in $\Delta$. In all other cases, we have

$$
\frac{1}{2} \rho_{\Delta}(\gamma)<\operatorname{Teich}_{\mathbb{K}}(H(\gamma))<\rho_{\Delta}(\gamma),
$$

for any non-constant, piecewise differentiable path $\gamma$ in $\Delta$.

Proof. Since we have already shown that $(1 / 2) \rho(p) \leq \lambda(p) \leq \rho(p)$, and since $\rho_{\Delta}$ is the lift to $\Delta$ of $\rho$ and $T e i c h \mathbb{K}$ is the lift to $\mathbb{K}$ of $\lambda$,

$$
\frac{1}{2} \rho_{\Delta}(\gamma) \leq \operatorname{Teich}_{\mathbb{K}}(H(\gamma)) \leq \rho_{\Delta}(\gamma) .
$$

Equality in (29) can hold only if the corresponding equality holds in

$$
(1 / 2) \rho(p) \leq \lambda(p) \leq \rho(p) .
$$

We have seen that $(1 / 2) \rho(p)=\lambda(p)$ if the component $R_{j}$ of $R$ that contains $p$ is simply connected. Conversely, if $R_{j}$ is not simply connected, then $R_{j}$ has a nontrivial function covering group $\Gamma$, and the quadratic differential

$$
\psi_{p}(z)=\sum_{\Gamma} \frac{\gamma^{\prime}(z)^{2}}{\pi \gamma(z)}
$$

has norm strictly less than 2. From (21) this implies that $\lambda(p)>(1 / 2) \rho(p)$.

It remains to show that if $R$ is not conformal to $\overline{\mathbb{C}} \backslash\{0,1, \infty\}$, then $\lambda(p)<\rho(p)$. We will prove this in the remainder of this section.

Theorem 10. If there is any point $p$ in $\overline{\mathbb{C}}-E$ for which $\lambda(p)=\rho(p)$, then $A(E \cup\{p\})$ is one dimensional and $E=\{0,1, \infty\}$.

Proof. First we show that the component of $R$ containing $p$ cannot be simply connected and it cannot be conformal to a punctured disc. Finally we show that if a component does not fall into one of these two cases, then it must contain a simple closed hyperbolic geodesic and whenever this is the case $\lambda(p)<\rho(p)$.

The first situation is excluded because we have already observed that if a component of $R$ is simply connected, then $\lambda(p)=(1 / 2) \rho(p)$ on that component.

In the second situation, we can assume the component of $R$ is equal to the punctured disc $\Delta^{\prime}=\{z: 0<|z|<1\}$ and that $0<p<1$. Consider the vector field $V(z) \frac{\partial}{\partial z}$ defined on $\Delta^{\prime}$ by

$$
V(z)=\frac{1}{p \ln p}(\ln |z|) z=\frac{1}{p \ln p} \cdot\left(\frac{\ln z+\ln \bar{z}}{2}\right) z .
$$


Notice that $V$ vanishes at 0 and on $\{z:|z|=1\}$, that $V(p)=1$ and finally that

$$
\mu=\bar{\partial} V=\frac{1}{2 p \ln p} \cdot \frac{z}{\bar{z}}
$$

Thus

$$
\lambda_{\Delta^{\prime}}(p) \leq\|\bar{\partial} V\|_{\infty} \leq \frac{1}{|2 p \ln p|}=\rho_{\Delta^{\prime}}(p) .
$$

But the expression in (32) is the Beltrami coefficient associated to the quadratic differential $q(z)=\frac{(d z)^{2}}{z^{2}}$ which over $\Delta^{\prime}$ is a quadratic differential of infinite norm. By Theorem 5 there is a unique quadratic differential holomorphic in $\Delta^{\prime}$ with the largest possible real residue at $p$ and with $L_{1}$-norm equal to one, which we denoted by $\varphi_{p}$. This linear functional $V$ is represented by the Beltrami coefficient $\lambda(p)\left|\varphi_{p}\right| / \varphi_{p}$. That is, any other Beltrami differential not of this form that represents $V$ must have larger $L_{\infty}$-norm. Since $\frac{(d z)^{2}}{z^{2}}$ is not integrable on $\Delta^{\prime}$, we conclude that $\mu$ in (32) does not have the smallest possible $L_{\infty}$-norm and so $\lambda(p)<\rho(p)$.

If $A(E \cup\{p\})$ is not one dimensional, then $E$ must contain four or more points. If the component $R_{j}$ of $\overline{\mathbb{C}}-E$ that contains $p$ is neither simply connected nor conformal to a punctured disk, then $R_{j}$ contains a simple closed curve homotopic to a Poincaré geodesic of positive length. Suppose that $\lambda(p)=\rho(p)$. Then by (16), $\|V\|_{T}=1$, where $V=H^{\prime}(0)$ and $H(t, z)=f^{\mu(t)}(z)$ is a holomorphic motion from Section 2. Thus, $\left\|\mu^{\prime}(0)\right\|_{\infty}=1$, and

$$
V_{0}=\left[\frac{\mu^{\prime}(0)}{\Pi^{\prime}(0)}\right]
$$

Formula (25) implies that

$$
V_{0}=\left[k \lambda(p) \frac{\left|\varphi_{p}\right|}{\varphi_{p}}\right],
$$

where $|k|=1$ and $\varphi_{p}$ is the differential in (26). Therefore,

$$
\frac{\mu^{\prime}(0)}{\Pi^{\prime}(0)}=k \lambda(p) \frac{\left|\varphi_{p}\right|}{\varphi_{p}} \text {. }
$$

Thus,

$$
\mu^{\prime}(0)=\tilde{k} \frac{\left|\varphi_{p}\right|}{\varphi_{p}}
$$

where $|\tilde{k}|=1$.

Let

$$
f(t)=\iint_{R} \mu(t) \varphi_{p} d x d y .
$$

Then $f$ maps $\Delta$ to $\Delta$ and

$$
f^{\prime}(0)=\iint_{R} \mu^{\prime}(0) \varphi_{p} d x d y=\tilde{k} .
$$

Thus, by Schwarz's lemma, $f(t)=\tilde{k} t$. We have

$$
\begin{aligned}
|t|=|\tilde{k} t| & =\left|\iint_{R} \mu(t) \varphi_{p} d x d y\right| \\
& \leq \iint_{R}|\mu(t)|\left|\varphi_{p}\right| d x d y \leq|t| \iint_{R}\left|\varphi_{p}\right| d x d y=|t| .
\end{aligned}
$$


Thus,

$$
\mu(t) \varphi_{p}=\tilde{k} t\left|\varphi_{p}\right|
$$

and $\mathbb{K}$ is a Teichmüller disc. The results in 11 or 4 now imply that $E$ is a three-point set.

Corollary 4. The ratio

$$
G([f])=\frac{\lambda(f(p))}{\rho(f(p))}
$$

is a well-defined real-valued function of $[f]$ in the Teichmüller space $T(E \cup\{p\})$ taking values between $1 / 2$ and 1 , and the extreme value $1 / 2$ only when $f(p)$ lies in a simply connected component of $f(R)$ and the extreme value 1 only when $f(R)$ is the complement of three points in the Riemann sphere.

\section{Local AND GLOBAL TeichmüLleR Densities}

In the proof of Theorem 10 we proved that $\lambda_{D}(p)<\rho_{D}(p)$ for the case that the domain $D$ is the punctured disc. Here we will give another method that can be applied to any domain. It involves the introduction of another density on $D$ which we will call the global Teichmüller density. We have seen that the density $\lambda(p)$ is equal to the Teichmüller norm of a vector field that is equal to zero on the boundary of $D$ and equal to 1 at $p$. In Section 2 we have also seen that the holomorphic motion $h$ in (10) has an extension to the whole complex plane. Now consider all possible extensions $\tilde{h}$ of $h$ to the whole plane and put

$$
V=\left.\frac{d}{d t}\right|_{t=0} \tilde{h}(t, z)
$$

and finally put

$$
\sigma(p)=\rho(p) \inf \left\{\|\bar{\partial} V\|_{\infty}\right\}
$$

where the infimum is taken over all such tangent vectors $V$ obtained as tangent vectors to holomorphic motions $\tilde{h}(t, z)$ of this type. Since $\sigma$ is obtained from the infimum over a subset of the vector fields used in the definition of $\lambda$, we obviously have the following inequality:

$$
\lambda(p) \leq \sigma(p)
$$

The inequality

$$
\sigma(p) \leq \rho(p)
$$

is obvious because $\sigma$ is given by the infimum in (33) and by Schwarz's inequality $\|V\|_{T} \leq 1$. We are calling $\sigma_{D}$ and $\lambda_{D}$ global and local because $\sigma_{D}$ depends on the class of vector fields that arise from global holomorphic motions defined for $|t|<1$ that do not alter the conformal structure of $D$, and the tangent vectors that determine $\lambda_{D}$ do not necessarily arise from such holomorphic motions.

There is also a corresponding metric which we call $\Sigma_{\mathbb{K}}(\tau, V)$ on the fiber $\mathbb{K}$. We can define it in the following way. Let $\tau$ be a point in $\mathbb{K}$ and consider the family $\mathcal{H}$ of all holomorphic maps $H$ from $\Delta$ into $\mathbb{K}$ with $H(0)=\tau, H^{\prime}(0)=V$ and with $F \circ H=\Pi$ in Figure 1. Let

$$
\Sigma_{\mathbb{K}}(\tau, V)=\inf \left\{\|\bar{\partial} \tilde{V}\|_{\infty}\right\}
$$

where the infimum is taken over all continuous extensions $\tilde{H}$ of the map $h$ in (10) with $\tilde{V}=\tilde{H}^{\prime}(0)$. 
From this definition it is obvious that for any point $\tau$ in $\mathbb{K}$ and any tangent vector $V$ at $\tau$,

$$
\operatorname{Teich}_{\mathbb{K}}(\tau, V) \leq \Sigma_{\mathbb{K}}(\tau, V) \leq K o b_{\mathbb{K}}(\tau, V) .
$$

We also have a proposition for $\sigma$ parallel to Propositions 1 and 2 for $\rho$ and $\lambda$.

Proposition 3. The $\Sigma$-density $\Sigma_{\mathbb{K}}([f], V)$ on $\mathbb{K}$ is related to the $\sigma$-density on $R_{j}$ by the formula:

$$
\sigma\left(F([f])|d F(V)|=\Sigma_{\mathbb{K}}([f], V),\right.
$$

where $F: \mathbb{K} \rightarrow R_{j}$ is the evaluation map $F([f])=f(p)$ in Figure 1 .

An alternative technique for showing that $\lambda(p)<\rho(p)$ relies on two observations. First, just as in the proof of Theorem 10, a vector field $V$ equal to 1 at $p$ and 0 on the boundary for which $\lambda(p)=\|\bar{\partial} V\|_{\infty}$ is unique and its $\bar{\partial}$-derivative must have Teichmüller form, that is, $\bar{\partial} V$ must be equal to

$$
k \lambda(p) \frac{\left|\varphi_{p}\right|}{\varphi_{p}},
$$

for some integrable holomorphic quadratic differential $\varphi_{p}$ and some number $k$, with $|k|=1$. Therefore, if we can find a vector field $V$ with $V(p)=1$ such that $\|\bar{\partial} V\|_{\infty} \leq$ $\rho(p)$ and such that $\bar{\partial} V$ is not of Teichmüller form, then necessarily

$$
\lambda(p)<\rho(p) .
$$

The second observation is that to find such a vector field $V$, instead of finding a formula for $V(z)$ on the whole plane as in the proof of Theorem [10, it suffices to find a holomorphic motion $H(t, z)$ that has the following properties:

(a) $H(t, z)$ extends $h(t, z)$,

(b) $H(t, z)$ is holomorphic for $|t|<1$,

(c) $H(t, z)$ is defined and holomorphic for $z$ in some nonempty open neighborhood $U$ contained in $D$, and

(d) for any $|t|<1, H(t, z)$ is injective in the variable $z$ on its domain of definition.

If $H(t, z)$ has these properties, then by Slodkowski's theorem it extends to a holomorphic motion $\tilde{H}(t, z)$ of $\overline{\mathbb{C}}$ and putting

$$
V=\frac{\partial}{\partial \bar{z}}\left(\left.\frac{d}{d t}\right|_{t=0} \tilde{H}(t, z)\right)
$$

by Schwarz's lemma $\|\bar{\partial} V\|_{\infty} \leq 1$. If we can do this, then since by part (c) $V$ is holomorphic in $U, V$ cannot be of Teichmüller form and therefore we conclude that $\lambda(p)$ is strictly less than $\rho(p)$.

To apply this approach to the punctured disc $D=\Delta^{\prime}=\{z: 0<|z|<1\}$ we create the partial extension $H(t, z)$ by decomposing the covering map $\Pi: \Delta \rightarrow \Delta^{\prime}$ in the following way. Let $T(w)$ be the Möbius transformation

$$
z=T(w)=\frac{w-i}{w+i}
$$

that transforms the upper half-plane onto the unit disc and let $f=\Pi \circ T$.

By normalizing $\Pi$ we may assume that $f$ is the universal covering from the upper half-plane given by the formula

$$
f(w)=\exp (2 \pi i w / c)
$$


where $c>0$ and the covering group is the cyclic group generated by the translation $w \mapsto w+c$. Since $f(i)=p$ we have $c=2 \pi / \log (1 / p)$. Let $W$ be a small open set with $p \in W$. Let $g$ be a branch of the inverse of $f$ for which $g(p)=i$ and let $U$ be the intersection of $W$ with $\{w:|w| \leq p\}$. The formula for $g(z)$ in $U$ is

$$
g(z)=\frac{c}{2 \pi i} \log z
$$

Now we define a partial extension of the motion $h(t, z)$ given in (10) by

$$
h(t, z)= \begin{cases}f\left(g(z)-i+T^{-1}(t)\right) & \text { for } z \in U \\ z & \text { for }|z| \geq 1 \text { and for } z=0 .\end{cases}
$$

Then $h(0, w)=f(g(w))=w$ for all $w \in U$ and the value of $h(t, z)$ remains the same if we replace $g(z)$ by another inverse branch of $f$. Also $h$ is holomorphic in $t$ and

$$
h(t, p)=f\left(g(p)-i+T^{-1}(t)\right)=f\left(T^{-1}(t)\right)=\Pi(t) .
$$

To check that $h$ is injective in $z$ assume

$$
f\left(g\left(z_{1}\right)-i+T^{-1}(t)\right)=f\left(g\left(z_{2}\right)-i+T^{-1}(t)\right) .
$$

That implies there is an integer $n$ such that

$$
g\left(z_{1}\right)-i+T^{-1}(t)=g\left(z_{2}\right)-i+T^{-1}(t)+n c .
$$

Therefore $g\left(z_{1}\right)=g\left(z_{2}\right)$ for $z_{1}$ and $z_{2}$ in $U$ and since $g$ is injective on $U$, that implies $z_{1}=z_{2}$.

By Slodkowski's extension theorem this $h$ can be extended to a motion of the whole plane. Any such extension will have tangent vector $V$ whose $\bar{\partial}$-derivative vanishes on $U$. Thus $V$ cannot be the extremal vector field that realizes the infimum in the definition of $\lambda(p)$ and so $\lambda(p)$ is strictly less than $\rho(p)$.

In general we can prove the following theorem.

Theorem 11. If $D$ is a simply connected domain, then

$$
\lambda_{D}(p)=\sigma_{D}(p)=\rho_{D}(p) / 2,
$$

if $D$ is conformal to $\mathbb{C} \backslash\{0,1\}$, then

$$
\lambda_{D}(p)=\sigma_{D}(p)=\rho_{D}(p),
$$

and for any other hyperbolic connected plane domain $D$,

$$
(1 / 2) \rho_{D}(p)<\lambda_{D}(p) \leq \sigma_{D}(p) \leq \rho_{D}(p) .
$$

Proof. Since these metrics are conformally invariant, to prove the first statement we only have to show that $\sigma_{\Delta}(0) \leq \rho_{\Delta}(0) / 2$, where $\Delta$ is the open unit disc. This inequality is realized by considering the holomorphic motion defined for $|t|<1$ by

$$
h(t, z)= \begin{cases}z+t(1-|z|) & \text { for }|z| \leq 1 \\ z & \text { for }|z| \geq 1\end{cases}
$$

Note that $h(t, z)$ is continuous, normalized to fix three points for all $|t|<1$ and fixes points $z$ in the exterior of the disc. To see that $z \mapsto h(t, z)$ is injective, first observe that for $|z| \leq 1,|h(t, z)| \leq|z|+|t|(1-|z|) \leq|z|+(1-|z|)=1$. Secondly, if $h\left(t, z_{1}\right)=h\left(t, z_{2}\right)$ and $\left|z_{1}\right|$ and $\left|z_{2}\right|$ are less than 1 , then

$$
z_{1}-z_{2}=t\left(\left|z_{1}\right|-\left|z_{2}\right|\right) \text {. }
$$


If $z_{1}$ is not equal to $z_{2}$ and $|t|<1$, this implies

$$
\left|z_{1}-z_{2}\right|<|| z_{1}|-| z_{2}||
$$

which is impossible, and we conclude that $z_{1}=z_{2}$.

To summarize, $h(t, z)$ is a holomorphic motion that fixes the boundary of the unit disc and $h(t, 0)=t$.

Finally, for $z$ in the interior of the unit circle

$$
V=\left.\frac{d}{d t}\right|_{t=0} h(t, z)=1-|z|=1-z^{1 / 2} \bar{z}^{1 / 2},
$$

and so

$$
\bar{\partial} V=-(1 / 2) \frac{z^{1 / 2}}{\bar{z}^{-1 / 2}}
$$

and the maximum value of $|\bar{\partial} V|$ in the unit disc is $1 / 2$, which implies $\sigma(p)=$ $(1 / 2) \rho(p)$.

The second statement of the theorem follows since we have already shown that in the case that $D=\mathbb{C} \backslash\{0,1\}, \lambda(p)=\rho(p)$, and the third statement is a consequence of Theorem 9 .

\section{ACKNOWLEDGEMENTS}

In preparing this manuscript we have benefited from helpful discussions with many people, especially Cliff Earle, Bill Harvey, Yunping Jiang, and Zhe Wang.

\section{RefERENCES}

[1] L. V. Ahlfors. Lectures on Quasiconformal Mapping,, volume 38 of University Lecture Series. Amer. Math. Soc., 2006. MR.2241787 (2009d:30001)

[2] L. Bers. An approximation theorem. J. d'Analyse Math., 14:1-4, 1965. MR0178287(31:2545)

[3] L. Bers. Fibre spaces over Teichmüller spaces. Acta Math., 130:89-126, 1973. MR0430318 $(55: 3323)$

[4] C. J. Earle. On holomorphic cross-sections in Teichmüller spaces. Duke Math. J., 36:409-416, 1969. MR0254233(40:7442)

[5] C. J. Earle and S. Mitra. Variation of moduli under holomorphic motions. Contemp. Math., 256:39-67, 2000. MR1759669 (2001f:30031)

[6] F. P. Gardiner. Approximation of infinite dimensional Teichmüller spaces. Trans. Amer. Math. Soc., 282(1):367-383, 1984. MR.728718 (85f:30082)

[7] F. P. Gardiner and N. Lakic. Quasiconformal Teichmüller Theory. Mathematical Surveys and Monographs, Vol. 76, Amer. Math. Soc., Providence, Rhode Island, 2000. MR.1730906 (2001d:32016)

[8] F. P. Gardiner and N. Lakic. Comparing Poincaré densities. Ann. of Math. (2), 154:247-259, 2001. MR1865971 (2003c:30046)

[9] F. P. Gardiner and N. Lakic. A vector field approach to mapping class actions. Proc. London Math. Soc., 92(3):403-427, 2006. MR2205723(2006k:30047)

[10] I. Kra. On the Thurston-Bers type of some self-maps of Riemann surfaces. Acta Math., 146:231-270, 1981. MR611385 (82m:32019)

[11] N. Lakic. Infinitesimal Teichmüller geometry. Complex Variables, 30:1-17, 1996. MR.1395227 (97i:30061)

[12] L. X. Liu and X. Yang. On some properties of infinite dimensional Bers fiber space. Northeast Math J., 15:203-208, 1999. MR1712183 (2000i:32024)

[13] S. Nag. Non-geodesic discs embedded in Teichmüller spaces. Amer. J. Math., 104:399-408, 1982. MR654412(83e:32027)

[14] H. Royden. Automorphisms and isometries of Teichmüller space. Annals of Math. Studies, 66:369-384, 1971. MR0288254(44:5452) 
[15] Z. Slodkowski. Holomorphic motions and polynomial hulls. Proc. Amer. Math. Soc., 111:347355, 1991. MR 1037218 (91f:58078)

[16] C. Zhang. On isomorphisms of Bers' fiber spaces. Ann. Acad. Scien. Fennica, 22:255-74, 1997. MR1469791 (98m:32034)

Department of Mathematics, Graduate School and University Center of CUny, New York, New York 10016 - and - Department of Mathematics, Brooklyn College, CUNY, BroOKLyn, New YORK 11210

E-mail address: frederick.gardiner@gmail.com

Department of Mathematics, Herbert H. Lehman College, Bronx, New York 10468

E-mail address: nlakic@lehman.cuny.edu 